\title{
Distinct solar wind pressure pulses producing convection twin-vortex systems in the ionosphere
}

\author{
D. V. Sarafopoulos \\ Department of Electrical and Computer Engineering, Demokritos University of Thrace, Xanthi, Greece \\ Received: 7 November 2003 - Revised: 10 February 2004 - Accepted: 25 February 2004 - Published: 14 June 2004
}

\begin{abstract}
We provide conclusive observational evidence demonstrating that a solar wind pressure pulse produces a twin-vortex system of ionospheric currents, while a stepwise pressure increase/decrease creates a single vortex structure, at high-latitude ground magnetograms. Multi-satellite (Wind, Geotail, Interball, IMP 8 and GOES 8) and multiinstrument observations of plasma and magnetic field confirm with in situ measurements that solar wind inherent pressure pulses and stepwise variations strike on the Earth's magnetosphere in a global scale. The ground signatures are scrutinized in detail and the twin- or single-vortex current systems are studied using the IMAGE array stations. One more finding of this work is that a single vortex can produce monopolar and bipolar features, depending on the station position relative to the overhead travelling convection vortex (TCV). All of the above observational evidence confirms Glassmeier's (1992) prediction model that associates solar wind's steep changes of pressure with ionospheric TCVs.
\end{abstract}

Key words. Magnetospheric physics (solar wind - magnetosphere interactions; current systems; magnetosphere - ionosphere interactions)

\section{Introduction}

Ground-based magnetometer arrays have been used for the study of travelling magnetospheric convection twin-vortices in the past. Such twin-vortex current systems have their vortex (TCV) centers separated by $1000-2000 \mathrm{~km}$, convect tailward with velocities $2.5-6 \mathrm{~km} / \mathrm{s}^{-1}$, and are located at high geomagnetic latitudes (Glassmeier, 1992; Lanzerotti et al., 1986; Glassmeier et al., 1989; Friis-Christensen et al., 1988; Heikkila et al., 1989; McHenry et al., 1990). The key feature of TCVs is an isolated magnetic field variation with a bipolar structure in the H-component, i.e. a negative-positive (NP) or positive-negative (PN) deflection of the Earth's magnetic field. The D-component associated with these H-component

Correspondence to: D. V. Sarafopoulos

(sarafo@ee.duth.gr variations comprises a single positive or negative excursion (Glassmeier, 1992).

Two physical mechanisms that generate such TCVs are extensively discussed in the past; the magnetic reconnection, and changes in the magnetopause pressure balance. Lanzerotti et al. (1986) discuss the possibility that TCVs are the ground signatures of flux transfer events at the dayside lowlatitude magnetopause. Glassmeier (1989) suggested examination of the reconnection process as the sudden switchon of a localized current in the magnetopause current region, which is oppositely directed to the magnetopause sheet current. The problems associated with the reconnection model are discussed in detail by Glassmeier (1992). FriisChristensen et al. (1988) have been able to correlate one of their twin-vortex events with a change in the solar wind dynamic pressure. Sibeck et al. (1989a, b) tried to argue, via schematic figures, that a step-like increase in the solar wind pressure probably gives rise to a double-vortex system.

Given that we adopt the solar wind abrupt pressure variations as the ultimate mechanism to generate TCV, then Kivelson and Southwood (1991), Glassmeier and Heppner (1992), and Lysak et al. (1994) have presented magnetohydrodynamic models that make conflicting predictions for event characteristics in the high-latitude ionosphere. Whereas the Glassmeier/Heppner model predicts that a transient compression of the magnetosphere should generate a pair of oppositely directed field-aligned currents (FACs) and vortices, the Lysak et al. model predicts that it will generate a triplet. The Kivelson/Southwood model predicts that a simple increase (or decrease) of pressure sets up a twin vortex configuration (dipolar source), while a pressure pulse gives rise to a quadrupolar source configuration and two current pairs, one pair at each end of the perturbed region (see also the review of Cowley, 2000). Kivelson and Southwood predicted that event amplitudes should peak on magnetic field lines that map to the abrupt density gradients at the inner edge of the low-latitude boundary layer (LLBL); Glassmeier and Heppner predicted that event amplitudes should peak on magnetic field lines that map to the magnetopause. In the model reported by Lysak et al. the events form at locations where field 


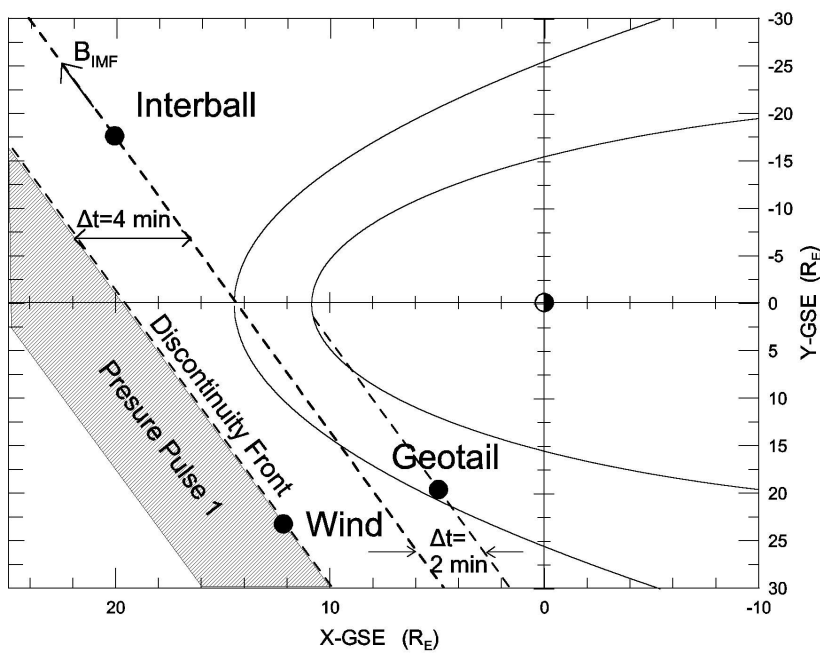

Fig. 1. Discontinuity front (thick-dashed line) for the solar wind pressure pulse (shaded area), which occurred on 3 July 1997, in three successive snapshots travelling toward the Earth. The average positions of the bow shock and magnetopause boundaries, as well as the positions of Interball, Wind and Geotail on the ecliptic plane, are shown.

line resonances occur. All the models concur to the point that the ionosphere response should be present at all local times, if the extent of applied solar wind pressure discontinuity along the Y-axis has a scale length similar to the magnetosphere's cross sectional dimension and, therefore, creates azimuthal pressure gradients all over the magnetosphere. In this paper we focus on those twin- and single-vortex ionospheric current systems for which their origin is strictly predetermined. We shall present three selected events characterized by three distinct solar wind pressure pulses and two isolated stepwise pressure variations that affect the Earth's ionosphere and ground magnetometer measurements. We observationally conclude that a solar wind dynamic pressure pulse will produce a system of twin TCVs over the IMAGE array stations, whereas a stepwise solar wind pressure variation will produce a single TCV. The possible directional change of the interplanetary magnetic field (IMF) does not play any significant role in the presented examples.

The carefully selected observations are not associated with the foreshock region and, therefore, are directly related to inherent solar wind pressure variations. We identify the solar wind pressure pulses and stepwise changes with multisatellite and multi-instrument plasma experiment moments and magnetic field measurements. With the multi-satellite observations upstream of the bow shock the extent and the orientation of the pressure discontinuity is firmly determined, so that one is persuaded that the whole magnetosphere is directly affected in a global scale. Therefore, we do not try to prove the global character of excited transient signatures using ground station magnetograms from more than one magnetometer arrays. We are based on the IMAGE array stations and associate their transient responses to the well-determined

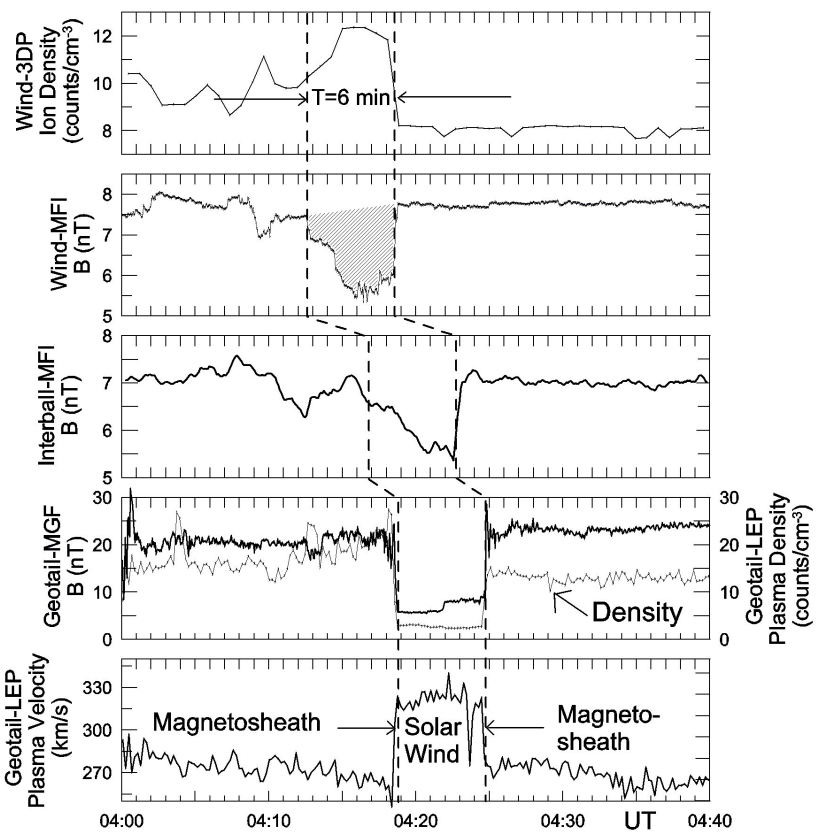

Fig. 2. The solar wind pressure pulse that occurred on 3 July 1997 , is confined between the two vertical dashed lines. The pulse apparently modulates the traces of magnetic field and plasma moment measurements obtained via the Interball, Wind and Geotail satellites. The magnetic field strength, the density and velocity are measured in nanoteslas, $\mathrm{cm}^{-3}$, and $\mathrm{km} / \mathrm{s}$, respectively.

pressure pulses. Certainly, although the selected events generally correspond to sudden impulses (SI) in lower-latitude ground magnetograms, we pay attention only to the highlatitude transient features.

The importance of our observations lies in the fact that until this time only one example has been published for which a directly detected solar wind pressure pulse produced a twinvortex structure (Friis-Christensen et al., 1988). Sibeck et al. (2003) demonstrated a case where pressure pulses are probably generated within the foreshock region and produced TCVs over the ionosphere. They used the geostationary GOES 6 and 7, as well as the IMP- 8 satellite in the foreshock region, and did not show solar wind inherent pressure pulses that affect the Earth's magnetosphere in a global scale. A case study of a storm sudden commencement (SC) that excites a twin-vortex ionospheric current system at high latitudes (Sastri et al., 2001) is rather relevant to the knowledge of the SC phenomenon itself. Therefore, we consider that it is out of the scope of this work to discuss further the possible similar responses in high-latitude stations caused by storm SC and solar wind pressure pulses.

In Sect. 2 the selected events (i.e. three pressure pulses plus two stepwise pressure drops) are exhibited. Ground signatures are interpreted and discussed in Sect. 3. 


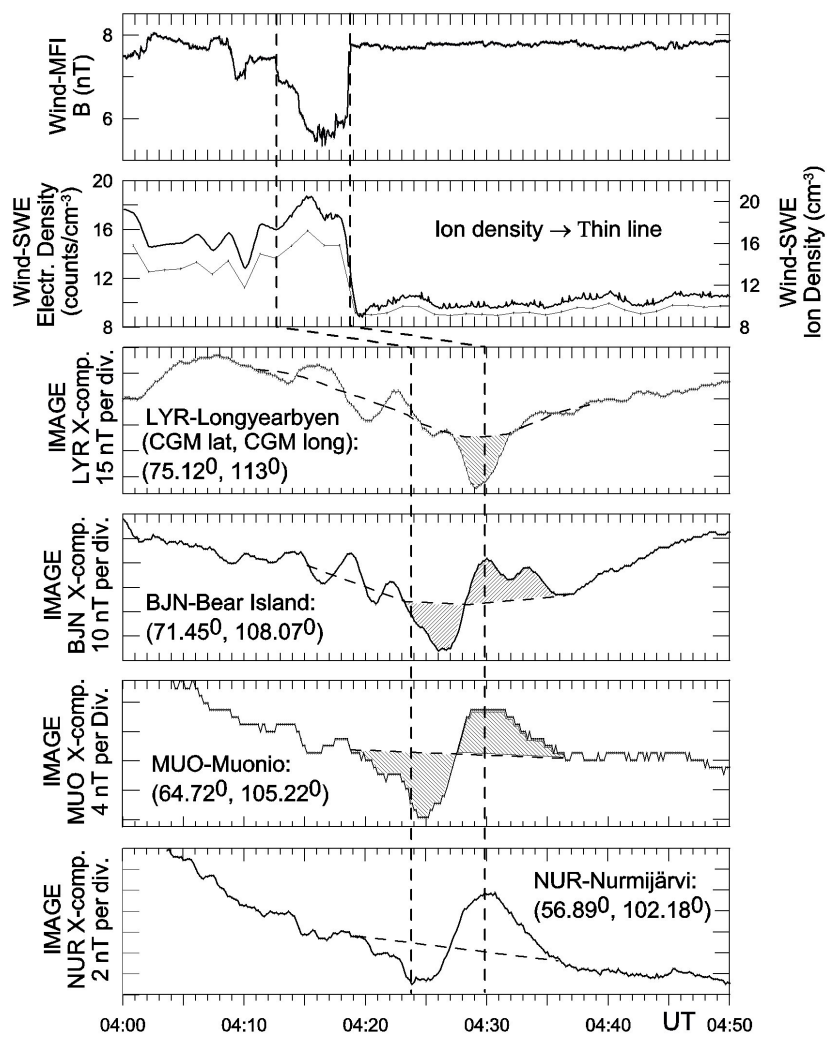

Fig. 3. The two vertical dashed lines define the $\sim 6$-min duration solar wind pressure pulse that occurred on 3 July 1997. Measurements obtained by the MFI magnetic field experiment, the 3DP and SWE plasma instruments on board Wind, and the associated ground signatures from the IMAGE array stations are shown from top to bottom. The pressure pulse produces bipolar features with progressively decreasing amplitudes at progressively lower CGM latitude stations. Along the highest latitude LYR station trace the periodic oscillations are mixed with the bipolar feature and only the negative excursion is very clear.

\section{Observations}

2.1 First (dawnside) event on 3 July (day 184) 1997:

Two successive solar wind pressure pulses

With this event a short solar wind pressure pulse lasting $\sim 6$ min seen by Wind and Interball pushed inward toward the magnetopause and the bow shock. Then, the Geotail satellite crossed the bow shock for almost the pulse duration interval. Figure 1 shows the satellite locations, the average bow shock and magnetopause positions, the pressure discontinuity front, the IMF direction during the pulse duration, as well as the extent of the first pressure pulse over the XY plane (shaded area). At 04:00 UT the Wind, Interball and Geotail satellites were located at $(\mathrm{X}, \mathrm{Y}, \mathrm{Z})_{G S E}=(12.57,23.2,-2.17)$, $(19.9,-17.4,6.34)$, and $(5.4,19.3,-0.25) R_{E}$, respectively. The positive pressure pulse is apparently imprinted along the Wind/3DP experiment ion density trace (Fig. 2, top panel, look at the interval in between thick-dashed vertical lines), as well as along the Wind/MFI and Interball/MFI magnetic
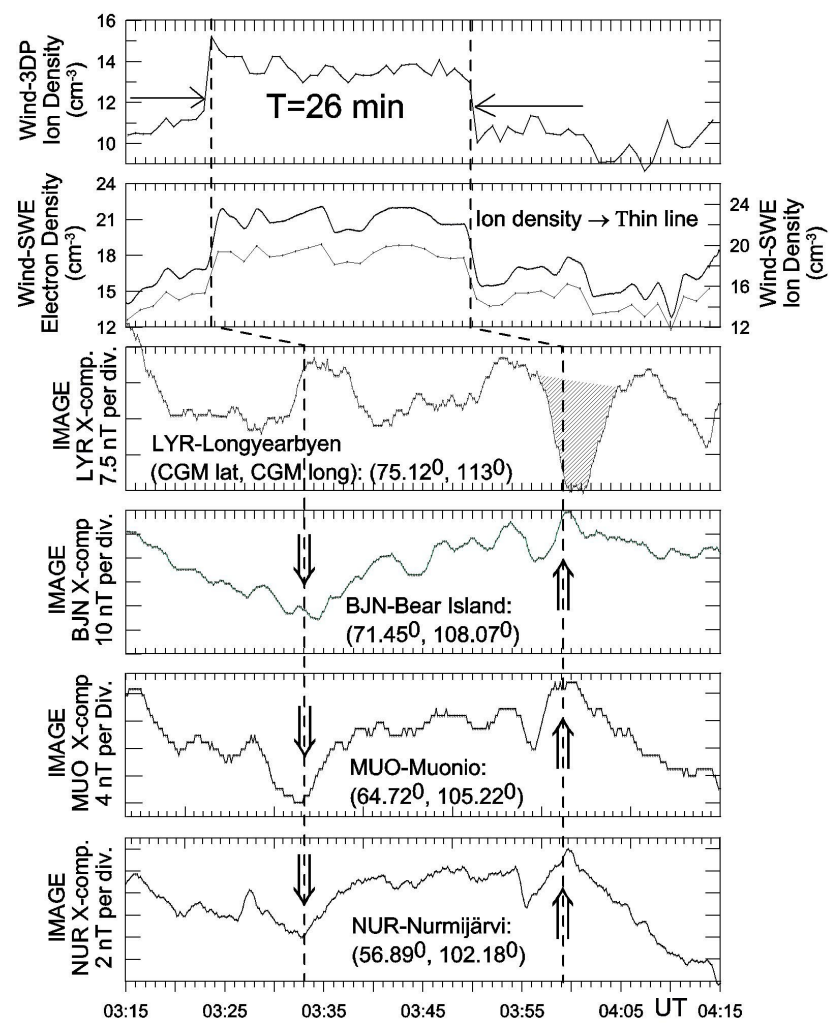

Fig. 4. Almost similar format as in Fig. 3 for the second longlasting pressure pulse episode. The bipolar feature is seen for all the IMAGE array stations, although with decreasing amplitude at decreasing latitudes. However, for the highest latitude LYR station only the negative excursion is clear.

field strength traces (Fig. 2, second and third panels). Additionally, it is reasonable to assume that the pressure enhancement diminished the dayside magnetosphere's radius, the magnetopause moved earthward and, consequently, Geotail was pushed for $\sim 6 \mathrm{~min}$ to abandon the magnetosheath proper crossing the bow shock. This latter spatial transition is observable along the magnetic field strength trace from the Geotail/MGF experiment, and the Geotail/LEP plasma velocity and density traces (Fig. 2, bottom two panels). Figure 1 shows the pressure discontinuity front in three successive snapshots travelling earthward. Note that it takes about 4 and $2 \mathrm{~min}$ for the discontinuity to be transported from Wind to Interball and from Interball to Geotail, respectively. Additional evidence about the solar wind pressure pulse carries the independent SWE experiment on board Wind.

The second panel in Fig. 3 shows the solar wind ion (thin line) and electron (thick line) densities, respectively, as measured by SWE. The pressure pulse produces clear bipolar features over the IMAGE array magnetograms. The negative-followed-by positive (NP-type) bipolar feature is apparently seen in the relatively lower latitude stations (i.e. Nurmijärvi-NUR, Muonio-MUO and Bear Island-BJN, three bottom panels). The same bipolar feature rather inverses at the highest latitude Longyearbyen-LYR station (third panel), 


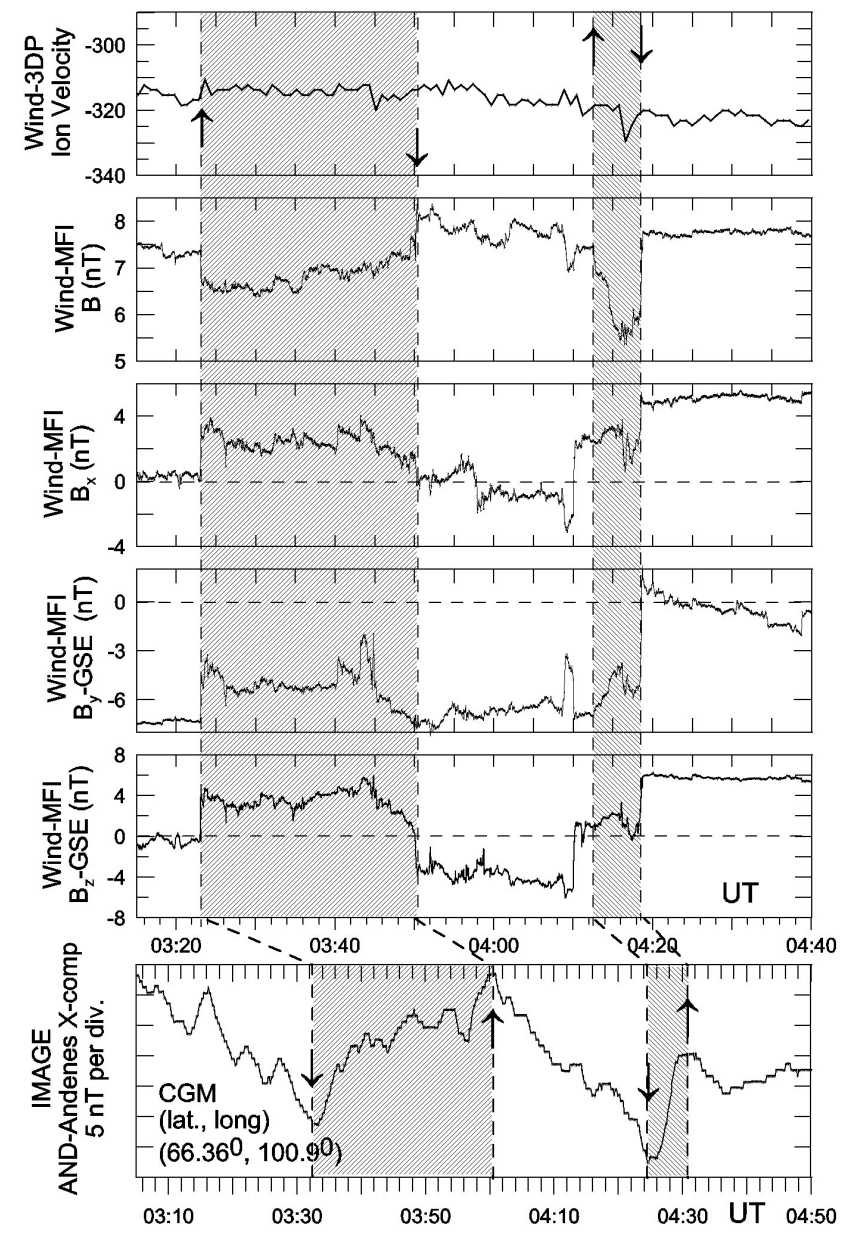

Fig. 5. The Wind spacecraft vector magnetic field measurements (MFI experiment) along with the ion plasma velocity (3DP instrument) and the ground Andenes (AND) station X-component trace (IMAGE array) throughout the interval of two successive solar wind pressure pulses (shaded areas) observed on 3 July 1997. The pulses do not change the pre-existing velocity level.

where we have to note that only the negative excursion (shaded area) is very clear.

A possible positive excursion is not clearly identified because of the always present periodic oscillations. The density increase (decrease) is associated with a negative (positive) excursion along, for instance, the NUR X-component trace. These isolated bipolar features, as we have stressed in the Introduction Section, are the key features for two TCVs in a twin-vortex system. In Corrected Geomagnetic Coordinates (CGM) the latitudes and longitudes of stations LYR, BJN, MUO and NUR are (75.12, 113.00), (71.45, 108.07), $(64.72,105.22),(56.89,102.18)^{\circ}$, respectively. The same sequence of bipolar features was identified $\sim 1 \mathrm{~h}$ ago, when another long-lasting pressure pulse this time $\sim 26$ min was observed. The reversed chronological order is dictated by the fact that the narrow pulse corresponds to the shaded area of Fig. 1 and is better determined by the two Geotail bow shock crossings. Figure 4 shows the long solar wind pressure pulse and the associated bipolar features on the ground as in Fig. 3. We determine the pulse duration by ion and electron density abrupt changes seen along the two independent Wind/3DP and Wind/SWE experiment traces (top two panels). Actually, the sequence of bipolar features seen by the LYR, BJN, MUO and NUR stations are similar to those analyzed in the previous short-pulse case. Certainly, for both exhibited pulse-cases we have to make clear that the density-time profiles are similar to those of solar wind dynamic pressuretime profiles. The latter is apparent in Fig. 5, where the solar wind velocity, as measured by the Wind/3DP experiment (top panel), essentially remained at the same level throughout the pulses' interval (shaded areas). Vector magnetic field measurements obtained by the Wind/MFI experiment are also included in Fig. 5. During the pulses the IMF strength was depressed (second panel), while the abrupt density variations were associated with directional changes in magnetic field. Most importantly, although both density decreases produce the same ground signature, they are associated with opposite $\mathrm{B}_{z}$ transitions: The short pulse was associated with positive and the long pulse with negative. Therefore, these $\mathrm{B}_{z}$ transitions seem to play down the hypothesis that the magnetopause reconnection causes the ground signatures and, in contrast, reinforce the consideration that they are produced directly by the pressure variations. At the bottom panel of Fig. 5 the X-component ground magnetogram from the Andenes (AND) station of IMAGE array is shown. The two successive bipolar features with negative/positive deflections (shaded areas) demonstrate similar ionospheric responses irrespective of pulse duration.

2.2 Second (dayside) event on 2 July (day 183) 1997: A negative pressure pulse followed by a step-like pressure drop

At 14:00 UT on 2 July 1997, upstream of the bow shock IMP-8, Wind and Geotail Satellites were located at (X, Y, $\mathrm{Z})_{G S E}=(35.7,-7.1,-6.5),(25.1,26.7,-3.5)$ and $(16.7,20$, $-1.51) R_{E}$, respectively. Figure 6 shows the three satellite positions, the IMF vector projected over the XY plane, and a snapshot from the convecting pressure pulse area (shaded region). The IMF points steadily dawnward (as we shall show later on) throughout the interval of study, 13:40-15:00 UT. A distinct negative solar wind pressure pulse at around $\sim 14: 00$ UT is seen by all three satellites: The density pulse is progressively detected along the IMP-8/PLA, Wind/3DP and Geotail/LEP plasma experiment measurements shown in the second, fourth and sixth panel traces of Fig. 7, respectively. The pressure pulse is associated with enhanced local magnetic field amplitude and positive excursion in $\mathrm{B}_{z}$ component. These magnetic field changes are qualitatively common for all three satellites, although indicative of only the amplitude variations for IMP-8 and Geotail (first and fifth panels), and the Wind $\mathrm{B}_{z}$ trace (third panel) are shown in Fig. 7.

A transient signature in the ground magnetic field seen by all the IMAGE network magnetograms is tightly associated 


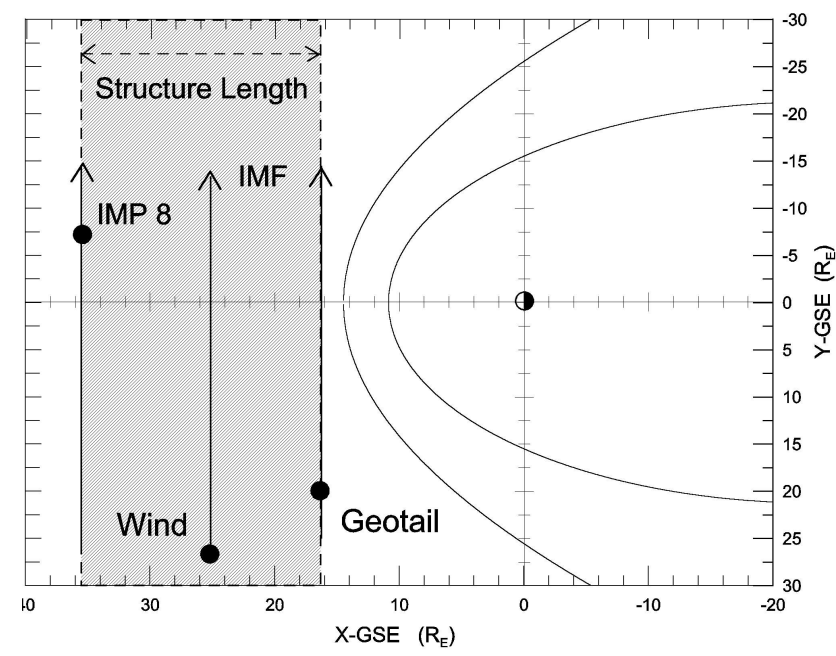

Fig. 6. A solar wind pressure pulse (shaded area) on 2 July 1997, convecting anti-sunward. IMP-8, Wind and Geotail record the upstream of the bow shock conditions. The satellite positions are projected on the $\mathrm{XY}_{G S E}$ plane.

with the solar wind pressure pulse. The bipolar PN-type structure is seen from the highest latitude stations like LYR, whereas the bipolar NP-type structure characterizes the lowest latitude stations like OUJ. The X-component traces from the just mentioned LYR and OUJ stations are indicatively shown in the bottom two panels of Fig. 7. These isolated bipolar features, as we explain in the Discussion Section, are due to two TCVs in a twin-vortex current structure. The isolated magnetic field variations and the well-defined solar wind pressure pulse classify this event as a classic "impulsive magnetic event" (Glassmeier, 1992). The solar wind pulse lasts $\sim 7 \mathrm{~min}$ and the whole structure upstream of the bow shock (shaded area in Fig. 6) has a length of $\sim 19 R_{E}$, which has been estimated using the solar wind velocity. The IMAGE station magnetograms are time-shifted $5 \mathrm{~min}$ to match the Geotail data, given that Geotail is the reference satellite. The drop in solar wind pressure increases the magnitude of the LYR station X-component, and after this enhancement the magnetic field does not return to its nominal value but decreases further to form a negative excursion. The sharp decrease in $\mathrm{X}$ is apparently caused by the backside increase of the solar wind pressure pulse. It seems that the monopolar positive excursion of pressure decrease combines with the monopolar negative excursion of pressure increase to produce the overall response in the LYR station. The duration of the bipolar feature on the ground is larger than the width of pressure pulse.

The Geotail vector magnetic field measurements, as well as the plasma velocity $\mathrm{V}_{X}$ and density from 13:40 to 15:00 UT are shown in Fig. 8. Actually, the density pulse (Fig. 8, top panel, shaded area) corresponds to a dynamic pressure pulse because the solar wind velocity has a constant value (second panel). During the under study period the IMF points dawnward (forth panel), the density is anticorrelated
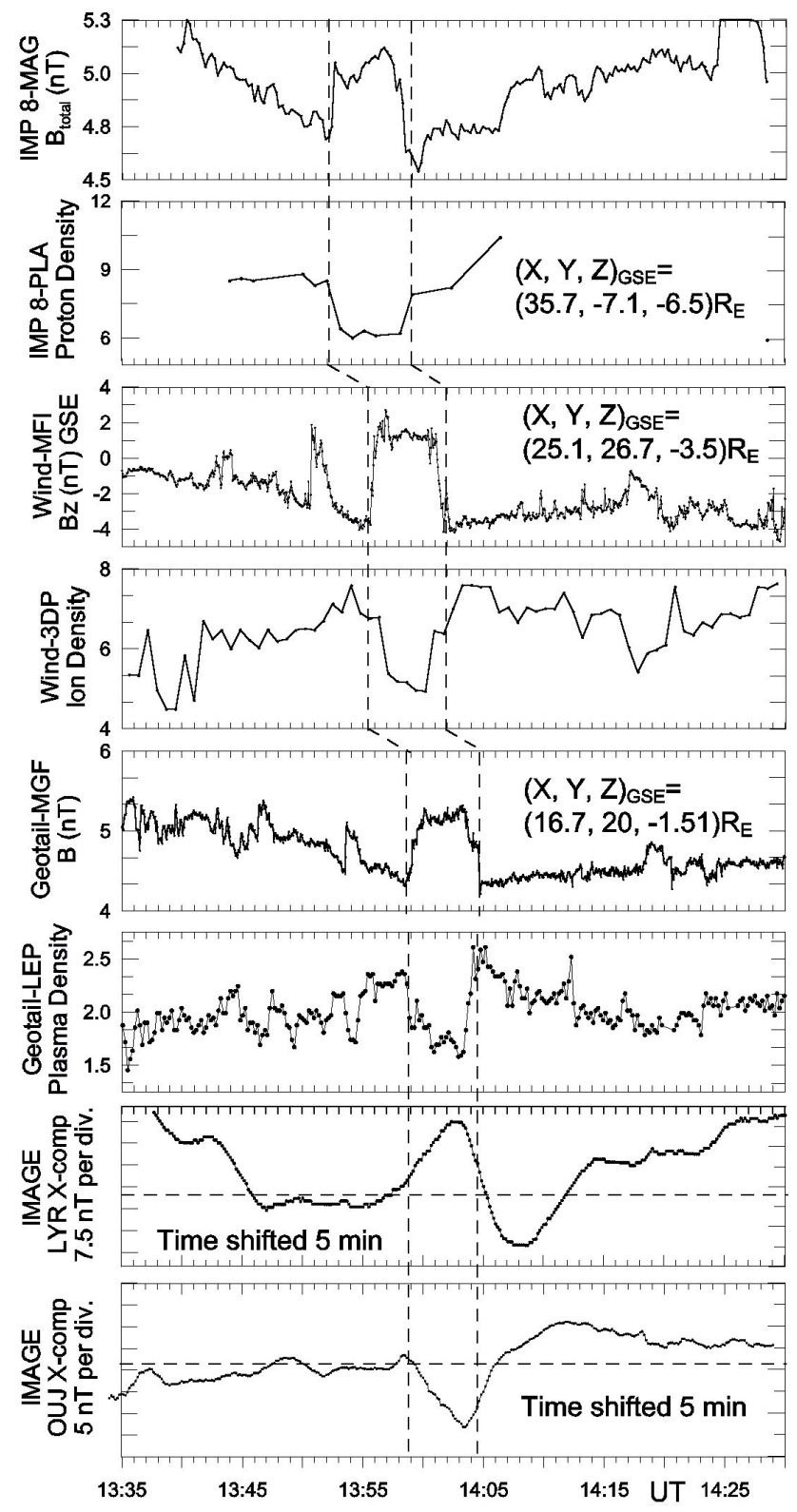

Fig. 7. From top to bottom, magnetic field (in nTs) and solar wind particle density (in $\mathrm{cm}^{-3}$ ) from IMP-8, Wind and Geotail. The two bottom panels show the ground signatures (PN- and NP-type for LYR and OUJ, respectively) produced by the negative solar wind pressure pulse, which is restricted between the two vertical dashed lines.

to the magnetic field strength (first and third panels) and, obviously, there is not any vector magnetic field connection between the pulse structure and the bow shock.

Half an hour later a stepwise solar wind density decrease is of great importance (Fig. 8, first panel). After the preceded study of three pressure pulses, one may anticipate monopolar features to accompany the step-like solar wind pressure drop. Actually, the latter is observationally confirmed in this case from the higher and lower latitude stations, although in a close' look, the mid-latitude IMAGE stations reveal a bipolar 


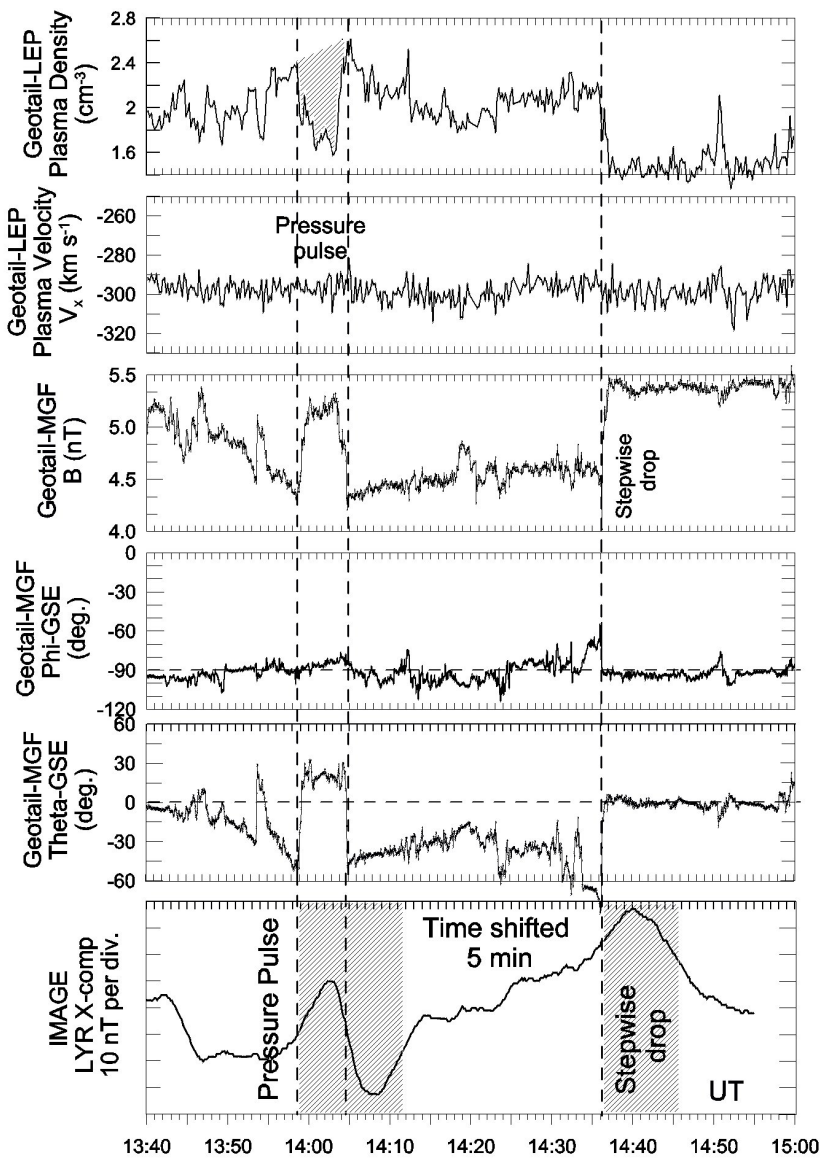

Fig. 8. Negative solar wind pressure pulse (top panel, shaded area) and stepwise pressure drop (single vertical dashed line) occurred on 2 July 1997. Measurements of plasma density and velocity as well as vector magnetic field from the Geotail plasma (LEP) and magnetic field (MGF) experiments are shown. The level of solar wind plasma velocity (second panel) is not affected by pressure variations. The ground LYR station X-component trace (from the IMAGE array) shows the characteristic signatures (shaded areas) corresponding to the pressure pulse and pressure drop.

feature. Figure 9 (third panel) shows the stepwise drop (increase) in Geotail solar wind plasma density (magnetic field magnitude) occurred at 14:36 UT. The same discontinuity was observed progressively earlier by Wind and IMP-8 at 14:33 and 14:28 UT, respectively. The $\mathrm{B}_{z}$ component of the IMF shows an abrupt increase (top two panels for IMP-8 and Wind) to almost zero value. The 3DP plasma experiment on board Wind reaffirms the abrupt drop in the ion density trace (second panel, thin line). The bottom three panels reveal the outstanding characteristics imprinted along the X-component traces and caused by the pressure change. The highest latitude LYR station shows a monopolar positive excursion, the mid-latitude BJN station shows a bipolar variation, and the lowest latitude OUJ station shows a monopolar negative excursion. The just presented X-component signatures give evidence for a single TCV, and the appropriate schematic figure is shown in the Discussion Section. The pressure pulse and

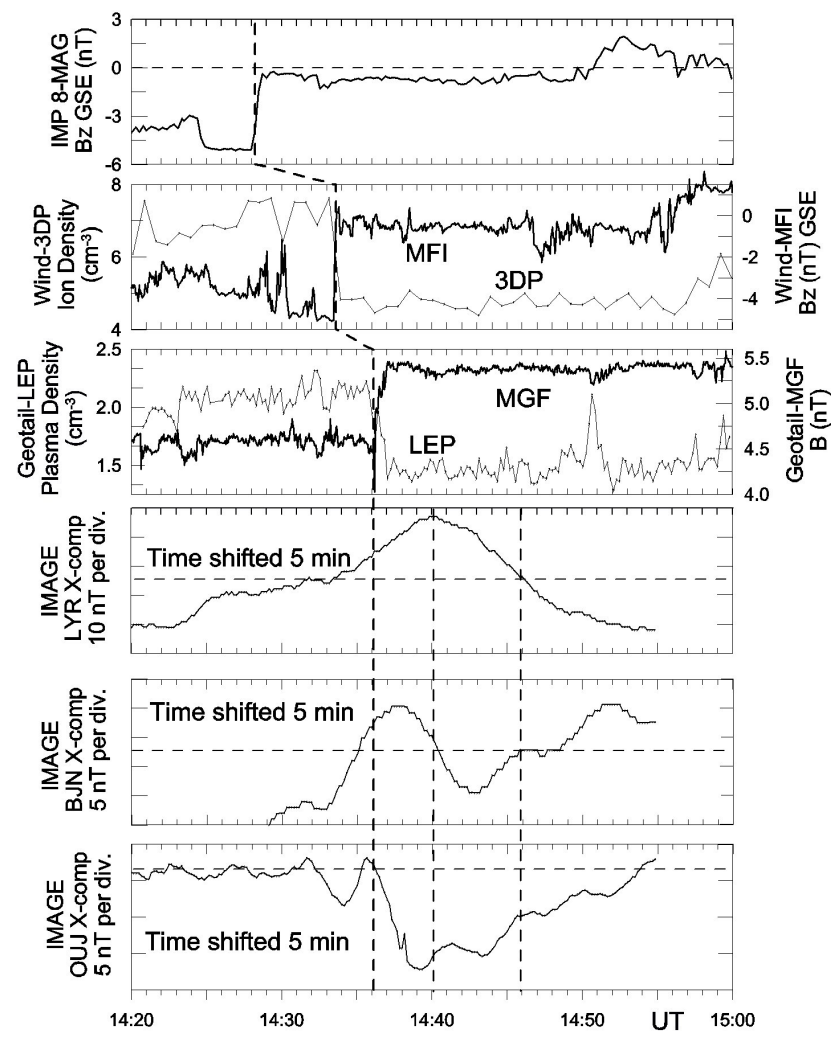

Fig. 9. Stepwise drop in solar wind pressure (vertical dashed line) identified along the magnetic field and particle density traces of data obtained by IMP 8 (top panel), Wind (second panel) and Geotail (third panel). The monopolar (positive in LYR station, negative in $\mathrm{OUJ}$ ) and bipolar (PN-type in BJN) ground signatures associated with the pressure variation are displayed in the three bottom panels.

pressure drop produce the features of shaded areas shown at the bottom panel of Fig. 8. It is worth noticing that the same highest latitude LYR station shows a PN bipolar feature under the influence of pressure pulse and, about $\sim 30$ min later, a positive monopolar feature produced by stepwise pressure drop. In the lowest latitude station (i.e. NUR) a NP bipolar feature is followed by a negative monopolar deviation (not shown here). We also note that after the applied pulse only the BJN station trace displays damping oscillations. In the next case study, we shall study an additional event characterized again by a stepwise decrease in solar wind density.

\subsection{Third (dayside) event on 7 August (day 219) 1997:}

Stepwise drop in solar wind pressure

All four widespread satellites in the solar wind plasma regime detect on 7 August, 1997, an abrupt stepwise increase (decrease) in magnetic field strength (solar wind plasma density). This variation occurred at 13:00 UT, when the Wind, Geotail, IMP-8 and Interball satellites were located at (X, $\mathrm{Y}, \mathrm{Z})_{G S E}=(105.2,-56.5,-15),(28.14,4.28,-1.9),(23.5$, $-29.5,-4.6)$ and $(7.5,-21.16,-6.73) R_{E}$, respectively. The spacecraft positions, the average magnetopause and bow shock boundaries, and the magnetic field discontinuity front 


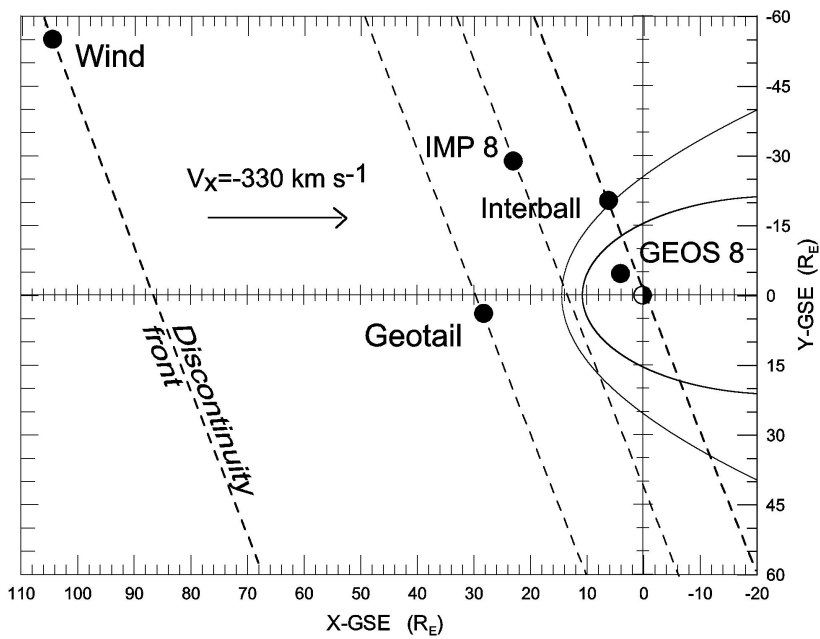

Fig. 10. The solar wind pressure discontinuity front that occurred on 7 August 1997, swept progressively the Wind, Geotail, IMP-8, GEOS-8 and Interball sites moving earthward. The satellite positions are shown projected on the ecliptic plane.

moving toward the Earth and projected over the XY plane, are shown in Fig. 10. The abrupt increase of the magnetic field strength was first detected by Wind at $\sim 12: 40$ UT (top panel, Fig. 11), and 19, 25 and 28 min later by Geotail, IMP8 and Interball, respectively (next three panels, Fig. 11). Shortly after the field discontinuity swept the Earth's magnetosphere and the ground-based stations recorded distinct transient deviations relatively to the average local magnetic field (bottom three panels, Fig. 11). The highest latitude NAL station shows a monopolar positive excursion, the midlatitude BJN station shows a bipolar PN-type variation, and the lowest latitude NUR station shows a monopolar negative excursion. These transient features, after a prolonged period without any perturbation, seem to be the result caused by the solar wind stepwise pressure drop. The latter is clearly evident by four autonomous plasma experiments (Fig. 12); the Wind/SWE electron density and the Wind/3DP ion density (first and second panels, thick line), the Geotail/CPI/SWA ion density (third panel), and the IMP 8/PLA proton density (bottom panel). Additionally, the ion solar wind velocities measured by Wind/3DP and Geotail/CPI/SWA are shown in the second (thin line) and forth panels, respectively. Throughout the event the ion solar wind velocity is essentially unvarying at the level of $\mathrm{V}_{x}=330-345 \mathrm{~km} / \mathrm{s}^{-1}$. The latter makes certain that the solar wind dynamic pressure time profile is similar to that of the plasma density characterized by the stepwise pressure drop. At $\sim 13: 05$ UT, this drop has a direct effect on the geostationary satellite GOES 8 magnetic field (Fig. 13, top panel). This moment GOES 8 was located at $(\mathrm{X}, \mathrm{Y}, \mathrm{Z})_{G S M}=(3.03,-5.9,0.08) R_{E}$ and actually, we have convincing evidence that the solar wind negative pressure jump strikes on the dayside magnetosphere at $\sim 13: 05$ UT. This time the GEOS $8 \mathrm{~B}_{z}$ component (Fig. 13, second panel) shows an abrupt decrease-decompression lasting $\sim 5 \mathrm{~min}$ and

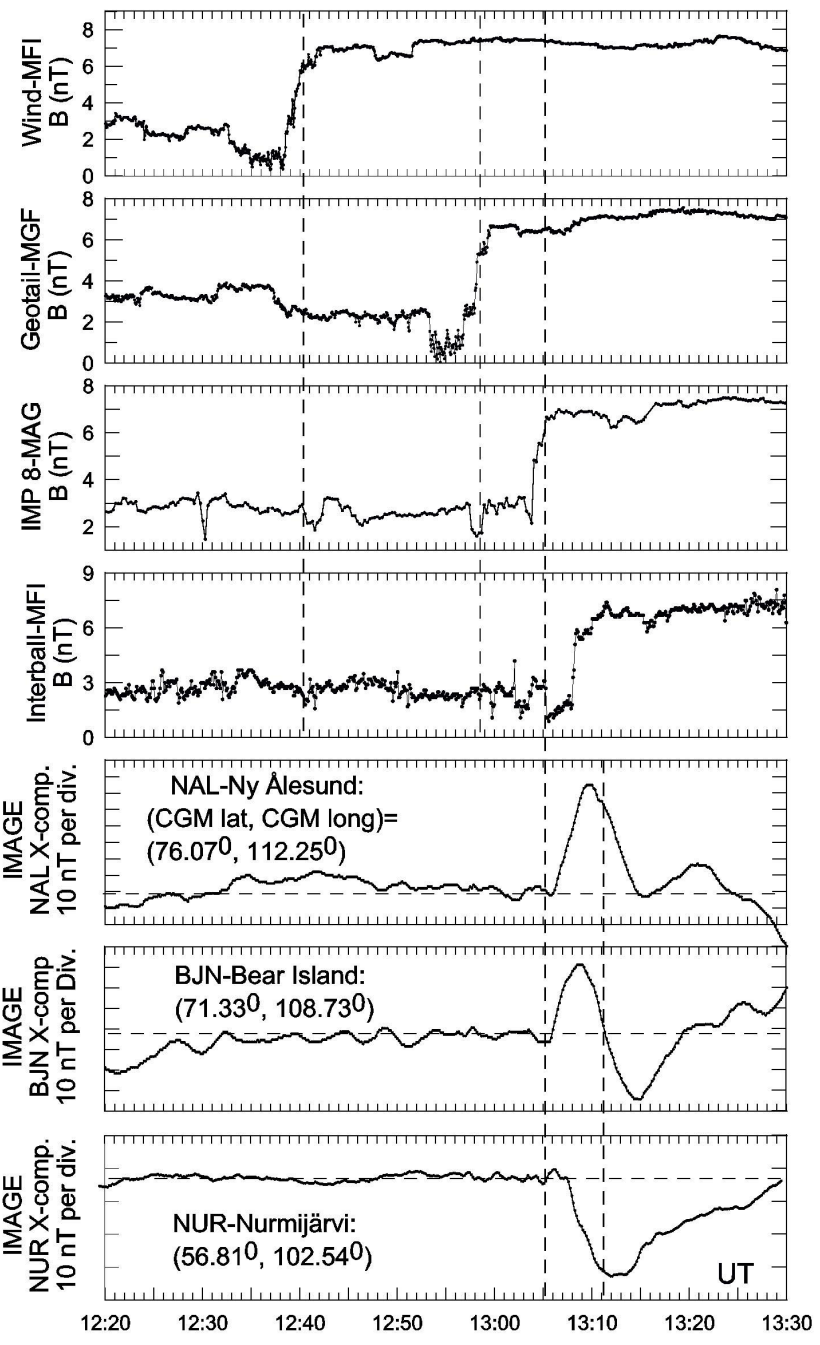

Fig. 11. Abrupt increases identified along the magnetic field strength traces of Wind, Geotail, IMP-8 and Interball are caused by the solar wind pressure drop that occurred on 7 August 1997. The discontinuity gives rise to monopolar positive (negative) excursion at NAL (NUR) station, and bipolar PN-type feature at BJN.

occurring simultaneously with the ground station responses (third panel). The GOES 8 measurements carry the information that a long lasting (i.e. more than $45 \mathrm{~min}$ ), gradual $\mathrm{B}_{z}$ component increase had been preceded the abrupt drop. After this drop in magnetic field strength, the magnetosphere seems to be switched to a relaxation state with a more dipolelike structure. Additionally, Fig. 13 (bottom three panels) shows the vector magnetic field measurements (magnitude, azimuthal and polar angles) for the IMF, as recorded by the Geotail/MGF experiment. Phi and theta angles (i.e. the $\mathrm{B}_{y}$ and $\mathrm{B}_{z}$ components) of the IMF change sign after the pressure drop because Geotail probably crossed an interplanetary current sheet. It is worth noticing that the stepwise pressure variation of this event was associated with the IMF transition to negative $\mathrm{B}_{z}$ values, whereas in the previous event the pressure drop was associated with a transition to positive $\mathrm{B}_{z}$. Nevertheless, for both $\mathrm{B}_{z}$ transitions the IMAGE 

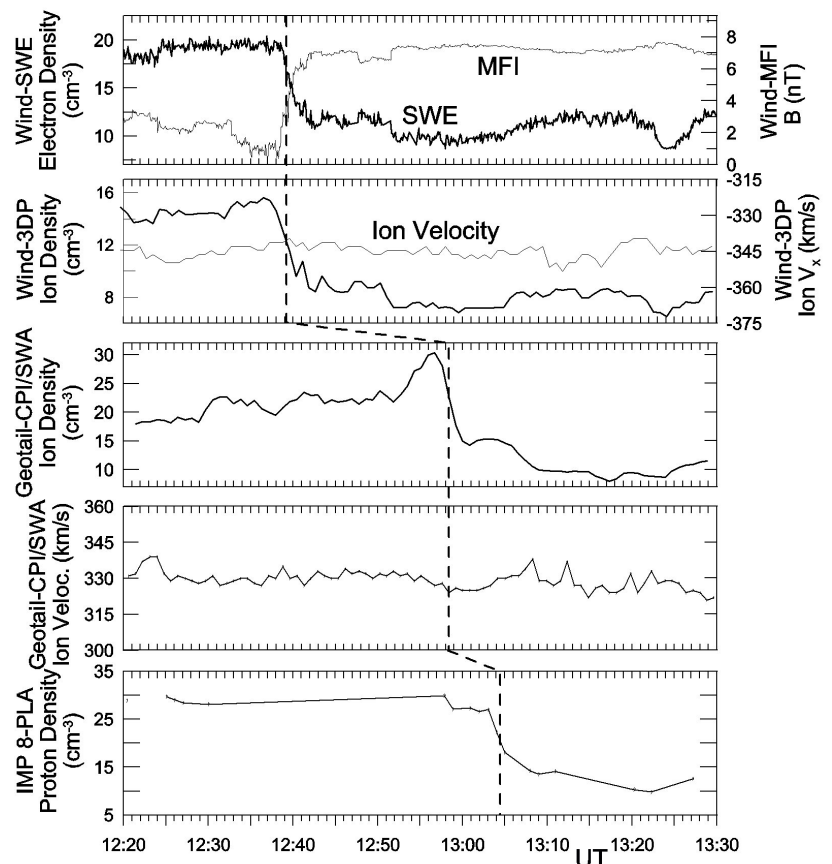

Fig. 12. The vertical dashed line defines the solar wind pressure drop that occurred on 7 August 1997. From top to bottom the Wind/SWE electron density (thick line) and the Wind/MFI magnetic field strength (thin line), the Wind/3DP ion density (thick line) and velocity (thin line), the Geotail/CPI/SWA ion density and velocity, and the IMP-8/PLA proton density are shown. The pressure variation does not change the solar wind velocity.

array stations show the same signatures. Certainly, the common and outstanding feature for both cases is the abrupt pressure drop, and not the directional changes in $\mathrm{B}_{z}$. Additionally, in the second case episode of stepwise pressure drop, the maximum peak-to-peak ground magnetometer variation is $\sim 80 \mathrm{nT}$, which is by far the strongest recorded during the interval 06:00-18:00 UT of the same day.

\section{Discussion}

\subsection{Interpretation of ground signatures}

From the three presented cases of twin TCVs, caused by solar wind pressure pulses, we infer that the high-latitude stations of IMAGE array display a bipolar feature, while the low-latitude stations show the inverse bipolar feature. This observation is interpreted through the schematic Fig. 14a, illustrating a system of twin TCVs moving antisunward above the IMAGE chain stations. Actually, the involved and drawn Hall currents will produce the anticipated features at the stations marked with the letters $\mathrm{A}$ and $\mathrm{C}$, which are representative of positions near the high- and low-latitude edges of vortices.

We also have presented two cases each of which demonstrates that a stepwise solar wind pressure drop produces monopolar features with opposite polarities at the high- and
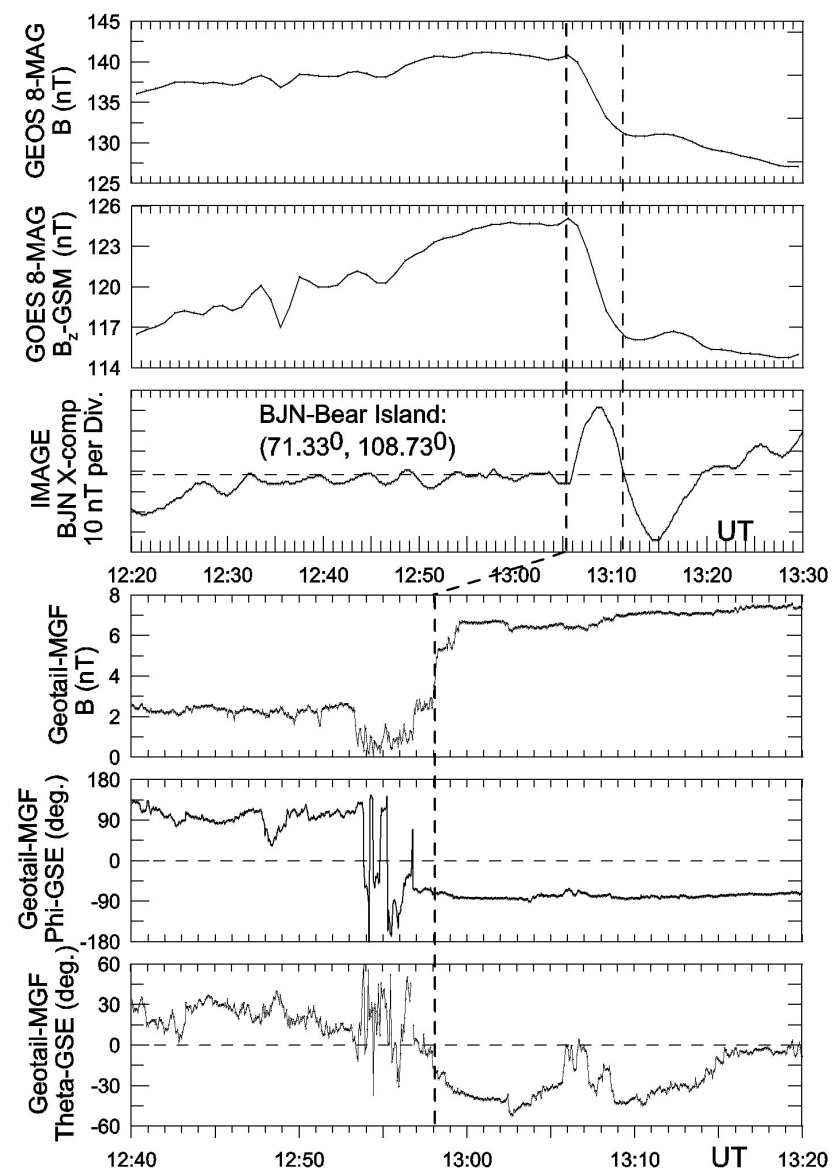

Fig. 13. The geosynchronous GEOS 8 satellite magnetic field magnitude, and $\mathrm{B}_{z}$ component, measurements concurrently with the BJN station X-component trace (three top panels). The steep decrease in $\mathrm{B}_{z}$ is associated with the PN-type feature in BJN station. Additionally, vector magnetic field measurements (strength, azimuthal and polar angles) obtained by Geotail are shown in the bottom three panels. The dashed line defines the time of solar wind pressure drop.

low-latitude IMAGE stations, and a bipolar feature at the mid-latitude stations. These observations are interpreted in the context of an antisunward and poleward moving vortex above the ground stations. Figure $14 \mathrm{~b}$ is a sketch illustrating the passage of a TCV with the associated Hall current through the ionosphere region that is in conjunction with the IMAGE stations. The North Pole points upward and the swirling ionospheric current produces a positive $\mathrm{X}$ component excursion at the high-latitude station marked with the letter A, a negative excursion at the low-latitude station $\mathrm{C}$, and a bipolar feature at the mid-latitude station $\mathrm{B}$. If the single vortex convects and the geomagnetic latitude remains constant, then only monopolar features would be anticipated. Therefore, the passage of a single TCV can produce either bipolar or monopolar features, depending on the station position relative to the above moving $\mathrm{TCV}$. 


\subsection{Reconnection and pressure pulses}

Flux transfer events (FTEs), or a reconnection process as the sudden switch-on of a localized current in the magnetopause current region are extensively discussed as candidate mechanisms generating single or twin-vortex structure (Glassmeier, 1992, and references therein). However, FTEs and reconnection are tightly associated with the $-\mathrm{B}_{z}$ component of the interplanetary magnetic field or transitions to $-\mathrm{B}_{z}$, which contradicts our observations. In the previous section, we have stressed the fact that the same ground signature is produced irrespective of the transition sign in $\mathrm{B}_{z}$. All three pressure pulse episodes produce ionospheric currents that are initiated with a $B_{z}$ jump to positive values. In one case, with solar wind stepwise pressure drop, the ground signature is associated with a positive sign in $\mathrm{B}_{z}$ transition. Most importantly, the reconnection model always predicts an upward current in the east and a downward current in the west, i.e. it always gives rise to bipolar ground signatures, if the current pair is convecting tailward. In contrast, our observations of stepwise pressure drops are associated with a single FAC, one vortex and a monopolar signature, even in the episode accompanied with transition to $-\mathrm{B}_{z}$ (Fig. 13). Therefore, we conclude that in our cases the dominant factor generating ionospheric vortices is the solar wind pressure pulses or stepwise variations. Certainly, we have not argued that all the ionospheric vortices that might be observed are excited by solar wind pressure gradients.

\subsection{Strong response at dayside}

A pressure discontinuity front perpendicular to the $\mathrm{X}$-axis bends as it encounters the bow shock because of refraction and becomes parallel to the magnetopause surface. Such bending occurs because the part of the wave nearest to the dayside magnetopause crosses the bow shock and slows first, while the wave at greater distances from the $\mathrm{X}$-axis continues forward at its full speed. The wave impact is mainly concentrated against the dayside magnetopause that is impacted by all three sides and the wave energy converges. If the discontinuity front in the solar wind does not move straight on, but at an angle toward the magnetosphere, then it will have a weaker impact. When a waveform crosses the bow shock, the speed and the wavelength diminish and the wave energy must increase the wave amplitude.

The consideration of the preceding paragraph probably explains why in the second and third studied events, which have occurred near noon local times, the ground magnetograms have demonstrated such an intense response (i.e. 40-80 nT). For both of them, the near noon local time stations observed the major responses, as compared to those recorded throughout the interval 06:00-18:00 UT of the corresponded day.

\subsection{Base Theory of this study}

We assume that any localized imbalance of pressure equilibrium at the magnetopause gives rise to a partial compression

\section{Twin Vortices convecting antisunward}
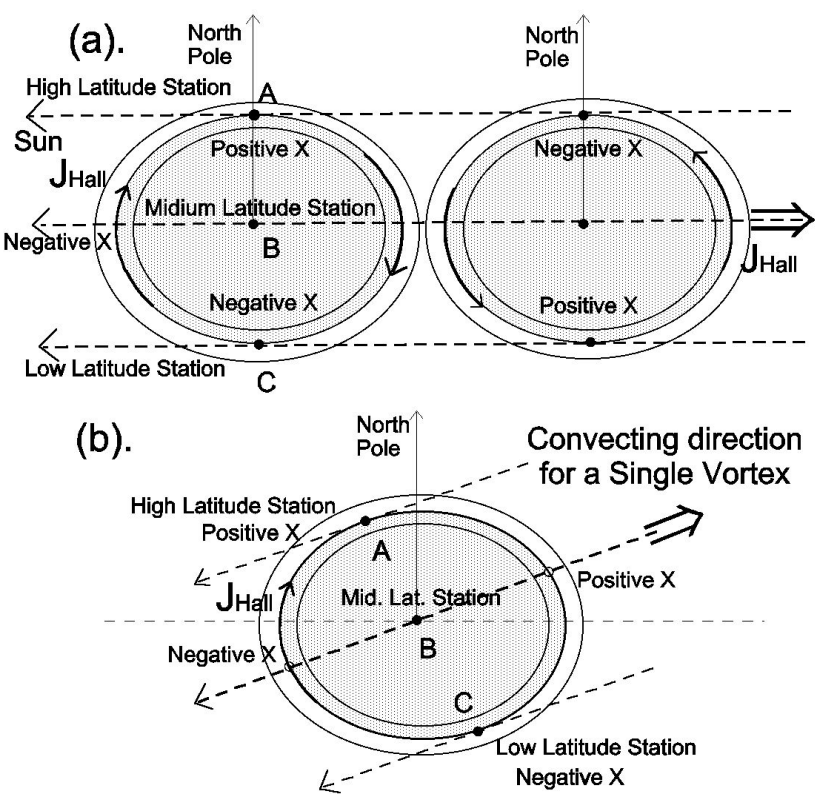

Fig. 14. (a) Twin vortex current system with opposite polarities of Hall currents moves antisunward above the IMAGE chain stations. Current flow is denoted with an arrow on each vortex structure, while the Sun is located to the left-side of this page. The high-(low)latitude station labelled A (C) is representative of positions near the high-(low-)latitude edges of vortices. The station A records an $\mathrm{NP}$ excursion along the $\mathrm{X}$-component magnetic field trace, while the station $\mathrm{C}$ records a $\mathrm{PN}$ excursion at the ground station $\mathrm{C}$. The medium latitude station $\mathrm{B}$ will not detect any $\mathrm{X}$-component variation. (b) Single vortex structure of Hall current convects antisunward and poleward, and produces a positive (negative) monopolar feature at station A (C), and a PN-type bipolar feature at station B.

or relaxation of the magnetosphere, which is accompanied by a change $\delta j_{M P}$ in the Chapman-Ferraro current system. The perturbation $\delta j_{M P}$ can be estimated from the momentum equation at the magnetopause

$\rho \frac{d \boldsymbol{v}}{d t}=-\nabla \delta p+\delta \boldsymbol{j}_{M P} \times \boldsymbol{B}_{0}$.

For steady solar wind plasma flow, or independent of time, we write $d v / d t=0$. The latter is actually our situation because the observed solar wind velocity did not change with the occurrence of pulse or stepwise solar wind pressure variations. For each of the three presented events this is apparently shown via Fig. 5 (first panel), Fig. 8 (second panel), and Fig. 12 (second and fourth panels). The inertial term according to Glassmeier and Heppner (1992) is always insignificant and may be neglected. The above equation gives

$\delta \boldsymbol{j}_{M P, \perp}=\frac{-\nabla \delta p \times \boldsymbol{B}_{0}}{B_{0}^{2}}$,

where the subscript " $\perp$ " denotes the field-transverse part of $\delta j_{M P}$. The inertial current has been neglected, while the right-hand side of Eq. (2) describes the diamagnetic currents 
associated with the pressure change. Presumably, $\delta j_{M P, \perp}$ partially closes via FACs that flow in the z-direction. In the same Cartesian coordinate system the $\mathrm{x}$-axis points outward along the magnetopause normal, and the y-axis points toward dusk tangential to the plane. The FAC density can be derived (Glassmeier and Heppner, 1992) from current continuity arguments

$$
\frac{\partial j_{F A C}}{\partial z}=\mu_{0} \frac{\partial \delta p}{\partial y} \frac{j_{M P}}{B_{0}^{2}} .
$$

Therefore, a single pressure gradient, $\partial \delta p / \partial y$, is associated with one single FAC and will produce one vortex. A positive pressure pulse is associated with the gradients $\partial \delta p / \partial y>0$ (upward FAC) and $\partial \delta p / \partial y<0$ (downward FAC, with respect to the north ionosphere), and will produce a twin-vortex system. Certainly this consideration agrees with our observations and confirms the predictions of Glassmeier and Heppner (1992). Sibeck et al. (2003) reached qualitatively similar results, although they did not drop the inertial term from Eq. (1). Kivelson and Southwood (1991) and Lysak et al. (1994) proposed current systems that contradict at least our observational results. The conclusion is that a solar wind pressure pulse will produce a pair of azimuthally spaced and oppositely directed FACs on field lines mapping to sharp pressure gradients at both the magnetopause and the inner edge of the low latitude boundary layer LLBL. A stepwise solar wind variation will produce one FAC.

\subsection{Additional comments}

For the three episodes of pressure variations observed at almost noon local times (second and third events), we infer that a pressure variation of $\sim 25 \%$ (second event) produces a maximum excursion along the X-component IMAGE array ground magnetograms $\sim 40 \mathrm{nT}$, while an $\sim 50 \%$ step pressure drop (third event) produces a maximum peak-to-peak ground bipolar feature $\sim 80 \mathrm{nT}$.

The study of magnetospheric ULF pulsations caused by solar wind dynamic pressure variations (for instance, look at Sarafopoulos, 1995; and Mishin et al., 2003) is disassociated from this research concerning isolated twin-vortex current structures. The latter was the fundamental selection criterion in this work. Intentionally, we have emphasized the long duration character $(\sim 25 \mathrm{~min})$ for the one pressure pulse that produces a twin-vortex system and ground signature, such as that of short pulses ( $\sim 6 \mathrm{~min})$.

We have not done a statistical work, however, on the base of direct correspondence between the solar wind abrupt pressure variations and the associated unique ground signatures; it is reasonable to infer that a solar wind pressure pulse (stepwise pressure variation) will always produce a twin-vortex (single vortex) structure at high-latitude stations. The determination of ground signatures caused by reconnection or flax transfer events is out of the scope of this work.

Each presented twin-vortex structure is generated by a solar wind pressure pulse, and each twin-vortex structure has to be associated with a pair of upward and downward flowing FACs. The FACs generate Alfvén waves and, therefore, the initial magnetoacoustic wave is transformed to an Alfvén wave. The observational support for a specific transformation mechanism (for instance like, the one proposed by Kozlovsky et al., 1994) may be the subject for future work.

\section{Conclusion}

The ultimate mechanism that produces twin- or single-TCV structures over the high-latitude ionosphere remains an issue under study. Moreover, there are models predicting different ground response-signatures caused by solar wind abrupt pressure variations. In this context, our presented events of TCV structures are uniquely due to solar wind pressure variations and, therefore, they are disassociated from any reconnection process over the dayside magnetopause. We have presented conclusive observational evidence that a distinct solar wind dynamic pressure pulse (stepwise pressure variation) produces a system of twin TCVs (single TCV) over the sub-auroral-polar stations. Additionally, it is evident from the analyzed cases that the pressure pulse response is equivalent to the response produced by two independent successive stepwise pressure changes with opposite sign.

One more finding is that the passage of a TCV can produce either a bipolar or a monopolar feature depending on the station position relative to the overhead moving TCV. All of the above evidence confirms the Glassmeier model prediction (1992) on the basis of which a solar wind pressure pulse is associated with two TCVs, and a stepwise variation to one TCV.

Acknowledgements. We are grateful to all Principal Investigators of the experiments MGF, LEP, and CPI/SWA of Geotail, MFI, 3DP and SWE of Wind, MFI of Interball, PLA and MAG of IMP 8, and MAG of GEOS 8. Concerning the use of ground magnetometer data, we are grateful to the Finnish Meteorological Institute (FMI/GEO), the Technical University of Braunschweig and other institutes that maintain the IMAGE magnetometer array. The author thanks both of referees.

Topical Editor T. Pulkkinen thanks D. Klimushkin and K. Takahashi for their help in evaluating this paper.

\section{References}

Cowley, S. W. H.: Magnetosphere-ionosphere interactions: A tutorial review, Magnetospheric current systems, AGU-Geophysical monograph 118, edited by Shin-ichi Ohtani, R. Fujii, M. Hesse and R. Lysak, 91-106, 2000.

Friis-Christensen, E., McHenry, M. A., Clauer, C. R., and Vennerstrom, S.: Ionospheric traveling convection vortices observed near the polar cleft: A triggered response to sudden changes in the solar wind, Geophys. Res. Lett., 15, 253-256, 1988.

Glassmeier, K. H.: ULF pulsations in the polar cusp and cap, in Electromagnetic coupling in the polar cusp and cleft, edited by Egeland, A. and Sandholt, P. E., Kluwer, Dordrecht, 167-186, 1989. 
Glassmeier, K.-H.: Traveling magnetospheric convection twinvortices: observations and theory, Ann. Geophysicae, 10, 547$565,1992$.

Glassmeier, K.-H., and Heppner, C.: Traveling magnetospheric convection twin vortices-Another case study, global characteristics, and a model, J. Geophys. Res., 97, 3977-3992, 1992.

Glassmeier, K. H., Hönisch, M., and Untiedt, J.: Ground-based and satellite observations of travelling magnetospheric convection twin-vortices, J. Geophys. Res., 94, 2520-2528, 1989.

Heikkila, W. J., Stockflet-Jorgensen, T., Lanzerotti, L. J., and MacLennan, C. G.: A transient auroral event on the dayside, J. Geophys. Res., 94, 15 291-15305, 1989.

Kivelson, M. G. and Southwood, D. J.: Ionospheric traveling vortex generation by solar wind buffeting of the magnetosphere, J. Geophys. Res., 96, 1661-1667, 1991.

Kozlovsky, A. E., Safargaleev, V. V., and Lyatsky, W. B.: The transformation of magnetoacoustic waves into Alfvén waves inside the magnetosphere, Ann. Geophysicae, 12, 1022-1026, 1994.

Lanzerotti, L. J., Lee, L. C., MacLennan, C. G., Wolfe, A., and Medford, L. V.: Possible evidence of flux transfer events in the polar ionosphere, Geophys. Res. Lett., 13, 1089-1092, 1986.

Lysak, R. L., Song, Y., and Lee, D. H.: Generation of ULF waves by fluctuations in the magnetopause position, in solar wind sources of magnetospheric ULF waves, Geophys. Monogr. Ser., vol. 81, edited by Engebretson, M. J., Takahashi, K., and Scholer, M., 273-281, AGU, Washington, D.C., 1994.
McHenry, M. A., Clauer, C. R., and Friis-Christensen, E.: Relationship of solar wind parameters to continuous, dayside, highlatitude travelling ionospheric convection vortices, J. Geophys. Res., 95, 15 007-15 022, 1990.

Mishin, V. V., Parkhomov, V. A., and Pashinin, A. Yu.: Geomagnetic pulsations caused by the magnetopause oscillations (comparison of spacecraft and geomagnetic observations), Adv. Space Res., 31, No 5, 1177-1182, 2003.

Sarafopoulos, D. V.: Long duration Pc5 compressional pulsations inside the Earth's magnetotail lobes, Ann. Geophys., 13, 926937, 1995.

Sastri, J. H., Takeuch, T., Araki, T., Yumoto, K., Tsunomura, S., Tachihara, H., Lühr, H., and Watermann, J.: Preliminary impulse of the geomagnetic storm sudden commencement of November 18, 1993, J, Geophys. Res., 106, p.p. 3905-3918, 2001.

Sibeck D. G., Baumjohann, W., Elphic, R. C., Fairfield, D. H., Gail, W. B., Fennell, J. F., Lanzerotti, L. J., Lopez, R. E., Lühr, H., Lui, A. T. Y., MacLeannan, C. G., McEntire, R. W., Potemra, T. A., Rosenberg, T. J., and Takahashi, K.: The magnetospheric response to 8 minute-period strong-amplitude solar dynamic pressure variations, J. Geophys. Res., 94, 2505-2519, 1989a.

Sibeck D. G., Baumjohann, W., and Lopez, R. E.: Solar wind dynamic pressure variations and transient magnetospheric signals, J. Geophys. Res., 16, 13-16, 1989 b.

Sibeck, D. G., Trivedi, N. B., Zesta, E., Decker, R. B., Singer, H. J., Szabo, A., Tachihara, H., and Watermann, J.: Pressure-pulse interaction with the magnetosphere and ionosphere, J. Geophys. Res., 108(A2), 1095, doi:10.1029/2002JA009675, 2003. 